\title{
Hyperthermic Intrathoracic Chemotherapy for Metastatic Ovarian Cancer
}

\author{
Annabelle Teng, MD, Barbara Wexelman, MD, Gary S. Schwartz, MD, Farr Nezhat, MD, \\ Gabriel Sara, MD, Scott Belsley, MD, Faiz Bhora, MD, Cliff Connery, MD \\ Department of Surgery, St. Luke's-Roosevelt Hospital Center, New York, NY, USA (Drs. Teng, Wexelman, Schwartz, Belsley). \\ Department of Obstetrics and Gynecology, St. Luke's-Roosevelt Hospital Center, New York, NY, USA (Dr. Nezhat). \\ Department of Hematology and Oncology, St. Luke's-Roosevelt Hospital Center, New York, NY, USA (Dr. Sara). \\ Department of Thoracic Surgery, St. Luke's-Roosevelt Hospital Center, New York, NY, USA (Drs. Bhora, Connery).
}

\begin{abstract}
Introduction: Ovarian serous carcinoma is an aggressive malignancy, often with metastases at presentation. Cytoreductive surgery combined with hyperthermic intraperitoneal chemotherapy has been studied as a treatment option for intraperitoneal spread of ovarian cancer. Hyperthermic intrathoracic chemotherapy, the thoracic counterpart to hyperthermic intraperitoneal chemotherapy, is emerging as a treatment option for both primary and secondary pleural cancers but has not previously been reported for treatment of ovarian metastases. The use of minimally invasive thoracoscopic techniques, including robotic assistance, for intrathoracic chemotherapy is rare. We present a case report of a patient with pleural metastasis of ovarian serous carcinoma treated with cytoreductive surgery and intrathoracic chemotherapy.
\end{abstract}

Case Description: In a 55-year-old woman who underwent a total abdominal hysterectomy and bilateral salpingooophorectomy with cytoreduction in July 2011 for ovarian cancer, a right-sided pleural effusion developed on postoperative radiographs. After chemotherapy treatment, she had a 7-month remission until positron emission tomography/ computed tomography scan identified an isolated pleural metastasis. The findings of diagnostic laparoscopy were negative for occult abdominal disease. We performed robotic-assisted right-sided video-assisted thoracoscopic surgery, partial pleurectomy, and resection of pleural, diaphragmatic, mediastinal, and pericardial nodules, followed by perfusion with heated cisplatinum for 60 minutes. The surgical procedure was uncomplicated, and the patient was discharged on postoperative day 3 . The serum creatinine level remained normal. The pathologic specimens showed poorly differentiated/high-grade adenocarcinoma consistent with the patient's ovarian primary. At 10 months postoperatively, she had no evidence of early recurrence and had a normal Karnofsky performance score (100).

Conclusion: We report the first case of robotic-assisted hyperthermic intrathoracic chemotherapy to treat ovarian carcinoma. Intrathoracic chemotherapy may be an important tool in the treatment of ovarian metastases in the chest.

Key Words: ovarian carcinoma, thoracic metastases, cytoreductive surgery, perfusion hyperthermic chemotherapy, robotic assistance

Citation Teng A, Wexelman B, Schwartz GS, Nezhat F, Sara G, Belsley S, Bhora F, Connery C. Hyperthermic intrathoracic chemotherapy for metastatic ovarian cancer. CRSLS e2014.00320. DOI: 10.4293/CRSLS.2014.00320

Copyright (C) 2014 SLS This is an open-access article distributed under the terms of the Creative Commons Attribution-Noncommercial-ShareAlike 3.0 Unported license, which permits unrestricted noncommercial use, distribution, and reproduction in any medium, provided the original author and source are credited.

Address correspondence to: Annabelle Teng, MD, Department of Surgery, St. Luke's-Roosevelt Hospital Center, 1000 Tenth Ave, Surgery Offices, Second Floor, New York, NY 10019, USA. Telephone: (212) 523-6970, Fax: (212) 523-6495, E-mail: anteng@chpnet.org

\section{INTRODUCTION}

Ovarian carcinoma is an aggressive malignancy that is often diagnosed with metastasis at presentation. Approximately 140000 women will die of ovarian cancer globally in 2014, and it is the fifth most common cause of death for women in the United States. ${ }^{1,2}$ The current standard treatment is complete cytoreductive surgery (CRS) followed by intravenous platinum-taxane chemotherapy alone or a combination of intravenous and intraperitoneal chemo- 
therapy. ${ }^{3-5}$ In view of the peritoneal surface of ovarian cancer, there is growing interest in the use of hyperthermic intraperitoneal chemotherapy (HIPEC) for women with recurrent epithelial ovarian carcinoma after surgical cytoreduction..$^{5-7}$ Hyperthermic intrathoracic chemotherapy (ITH), the thoracic counterpart to HIPEC, is a developing treatment modality for both primary and secondary pleural cancers but has not previously been reported for the treatment of ovarian metastases.

Intracavitary chemotherapy ${ }^{8}$ is advantageous over traditional intravenous chemotherapy administration in that an increased surface area of the tumor cells is exposed to cytotoxic agents with less systemic toxicity. Chemotherapeutic agents, however, can penetrate a depth of only a few millimeters. Therefore CRS is a prerequisite for intracavitary chemotherapy because maximum macroscopic tumor removal creates the optimal exposure to the cytotoxic agents. Finally, hyperthermia itself is known to be cytotoxic by causing impaired DNA repair, denaturation of proteins, and induction of apoptosis. ${ }^{8}$ The cytotoxic effect of hyperthermia is dependent on the time of heat exposure to the tissues and time relation to other therapies in addition to temperature. Hyperthermia also increases cell membrane permeability, thus increasing drug uptake by tumor tissue. This synergism can occur at mild hyperthermic temperatures $\left(39^{\circ} \mathrm{C}-41^{\circ} \mathrm{C}\right)$ with drugs such as cisplatinum and mitomycin $\mathrm{C}$ but has been observed to decrease at temperatures $>43^{\circ} \mathrm{C}$. Small bowel heat toxicity has also been shown to increase with temperatures $>$ $43^{\circ} \mathrm{C}$. Moreover, ITH is limited to a maximum temperature of $43^{\circ} \mathrm{C}$ because of an increased risk of pulmonary edema above this temperature. ${ }^{9}$

We present a patient with pleural metastasis of ovarian serous carcinoma treated with CRS and ITH by roboti- cally assisted minimally invasive thoracoscopic techniques.

\section{CASE REPORT}

The patient is a 55-year-old woman who underwent a total abdominal hysterectomy and bilateral salpingo-oophorectomy with cytoreduction in July 2011 for ovarian cancer and was noted to have a right-sided pleural effusion on postoperative radiographs. A thoracentesis showed that the effusion contained malignant cells. The patient completed an intravenous adjuvant chemotherapy regimen in March 2012 and remained in remission until a follow-up positron emission tomography/computed tomography (CT) scan in November 2012 showed an isolated right-sided pleural metastasis (Figure 1). She sought consultation at our institution, where she underwent a diagnostic laparoscopy 2 months later, which did not identify occult peritoneal disease. Because of a questionable history of platinum allergy, the patient was given 1 cycle of intravenous platinum as a test dose 3 weeks before a planned intrathoracic CRS and hyperthermic chemotherapy perfusion.

\section{Procedure}

The patient underwent right-sided video-assisted thoracoscopic surgery with robotic assistance, as well as partial pleurectomy with resection of pleural, diaphragmatic, mediastinal, and pericardiac metastatic nodules. She was intubated with a single-lumen endotracheal tube with a bronchial blocker under bronchoscopic guidance to achieve one-lung ventilation. A portal approach was used without an access incision, similar to our setup for roboticassisted lobectomy. This entailed 5 incisions: two 8-mm ports for robotic arms, a 5-mm thoracic grasper port, a
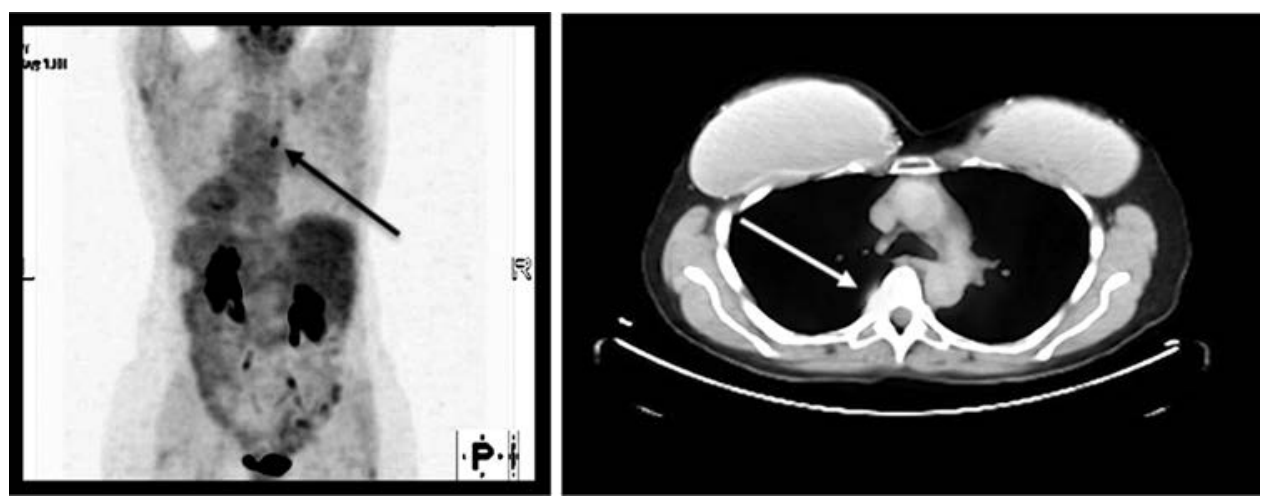

Figure 1. Follow-up positron emission tomography/CT scan. Nine months after the completion of the adjuvant chemotherapy regimen, the patient was found to have an isolated right-sided pleural metastasis (arrows). 

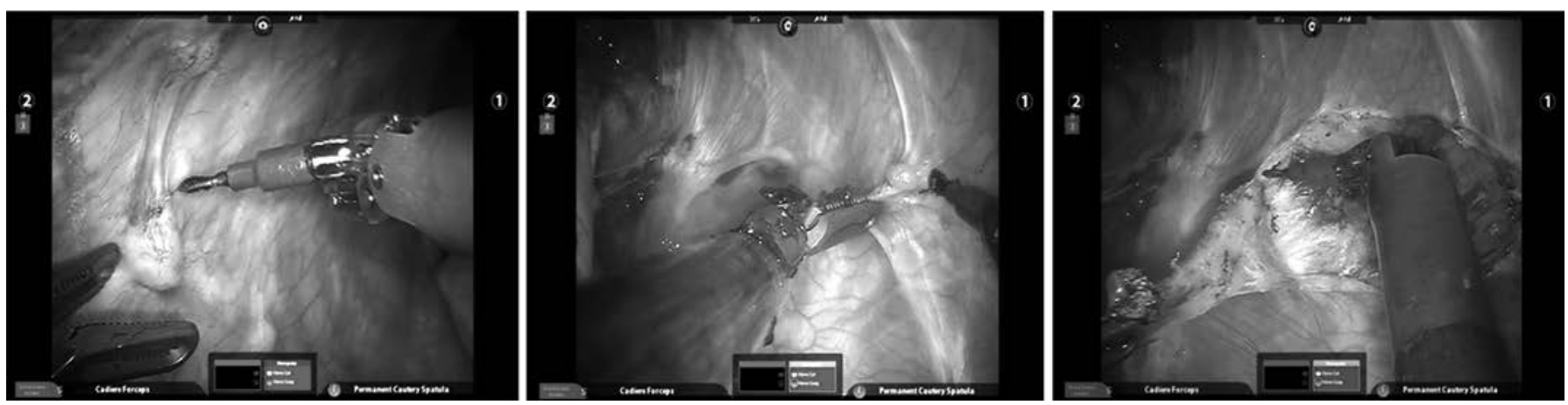

Figure 2. Cytoreductive surgery, comprising robotic-assisted right-sided video-assisted thoracoscopic surgery with partial pleurectomy and resection of pleural, diaphragmatic, mediastinal, and pericardiac metastatic nodules.

12-mm camera port, and a 15-mm working/specimen retrieval port.

The inflammatory adhesions were lysed, and examination of the pleura showed that although no significant pleural fluid or visceral pleural nodules were present, there were scattered parietal pleural nodules as expected. The largest lesion was $0.8 \mathrm{~cm}$ thick and $5 \mathrm{~cm}$ in diameter, was located in the posterior mid thorax adjacent to the head of the ribs, and was resected along with all other visible nodules (Figure 2). Robotic assistance allowed for maximum mobility and angulation to reach the lesions on the pleural surface. Larger lesions were resected individually, whereas smaller lesions were resected with partial pleurectomy by use of monopolar and bipolar cautery. Tumor nodules in the pericardial fat were resected by sharp dissection with hemostasis achieved with robotic clips and bipolar cautery. All visible macroscopic disease was resected. The patient was then prepared for hyperthermic chemoperfusion after hemostasis was ensured.

\section{Chemoperfusion}

Two inflow catheters were placed in the right and left robotic arm trocar sites, with one cannula just above the diaphragm and the other more anterior within the thorax positioned more cephalad. A 32F channel drain acting as the drainage cannula was placed through the camera port site, and the remainder of the working port sites were closed with sutures. The cannulas were connected to the Thermachem perfusion device circuit (Eight Medical, St. Paul, Minnesota). The reservoir had been preheated with Ringer lactate solution, which was used to fill the thorax. While the cisplatinum chemoperfusate was being prepared, the anesthesiologist administered $1503 \mathrm{mg}$ (910 $\mathrm{mg} / \mathrm{m}^{2}$ ) of amifostine intravenously over a 15 -minute period for renal protection. ${ }^{10}$ ITH was performed with 330



Figure 3. Postoperative chest radiographs. The chest tubes are in the right-sided pleural space with reinflation of the lung.

$\mathrm{mg}\left(200 \mathrm{mg} / \mathrm{m}^{2}\right)$ of cisplatinum diluted with normal saline solution with flow rates between 1200 and $1700 \mathrm{~mL} / \mathrm{min}$ for 60 minutes. Inflow temperatures were maintained between $41.3^{\circ} \mathrm{C}$ and $42.4^{\circ} \mathrm{C}$, and outflow temperatures were maintained at $41^{\circ} \mathrm{C}$ to $42^{\circ} \mathrm{C}$. The patient's systemic temperature at the start of perfusion was $34.6^{\circ} \mathrm{C}$. External cooling with cooling blankets and icepacks was used during the perfusion to control the core body temperature. After 60 minutes, the cisplatinum was drained and the thorax was flushed with $3 \mathrm{~L}$ of Ringer lactate solution. Sodium thiosulfate was then infused intravenously for additional renal protection, initially with a bolus of $7 \mathrm{~g}$ ( 4 $\mathrm{g} / \mathrm{m}^{2}$ ) over a 10 -minute period, followed by $20 \mathrm{~g}$ (12 $\mathrm{g} / \mathrm{m}^{2}$ ) over the next 6 hours. ${ }^{10}$

At the conclusion of the procedure, the patient's core body temperature was $36.5^{\circ} \mathrm{C}$. Excellent urine output was maintained throughout the perfusion, and an OnQ catheter (I-FLOW, Lake Forest, California) for postoperative pain management was placed under direct thoracoscopic vision. A $28 \mathrm{~F}$ angled chest tube was placed over the 
diaphragm, and the lung was reinflated (Figure 3). All remaining incisions were closed with sutures, and the patient was extubated at the end of the case. She was transferred to the surgical intensive care unit for observation.

\section{RESULTS}

The pathologic samples showed metastatic poorly differentiated/high-grade adenocarcinoma consistent with the patient's known primary of ovarian serous carcinoma. The surgical procedure was uncomplicated, and the patient tolerated the procedure well. She was observed in the surgical intensive care unit for 1 day and was discharged home on postoperative day 3 . The serum creatinine level remained in the normal range at 0.7 $\mathrm{mg} / \mathrm{dL}$, and the serum cisplatinum level postoperatively was $1500 \mu \mathrm{g} / \mathrm{L}$ without concern regarding toxicity. There was no evidence of recurrence by whole-body positron emission to-
mography/CT at 3 months after surgery (Figure 4). A subsequent CT scan of the chest, abdomen, and pelvis obtained at 8 months showed no evidence of disease recurrence. At 10 months, the Karnofsky performance score was 100 with no clinical signs of recurrence.

\section{DISCUSSION}

Current research in the surgical management of ovarian cancer is focused on the added benefits of HIPEC. ${ }^{5-7}$ Although ITH has been studied for the treatment of pleurabased cancers including mesothelioma and metastases from thymic carcinoma, there are no published reports of the use of ITH in cases of pleural metastases from ovarian carcinoma.

The minimally invasive approach minimizes morbidity and contributes to a decreased hospital length of stay
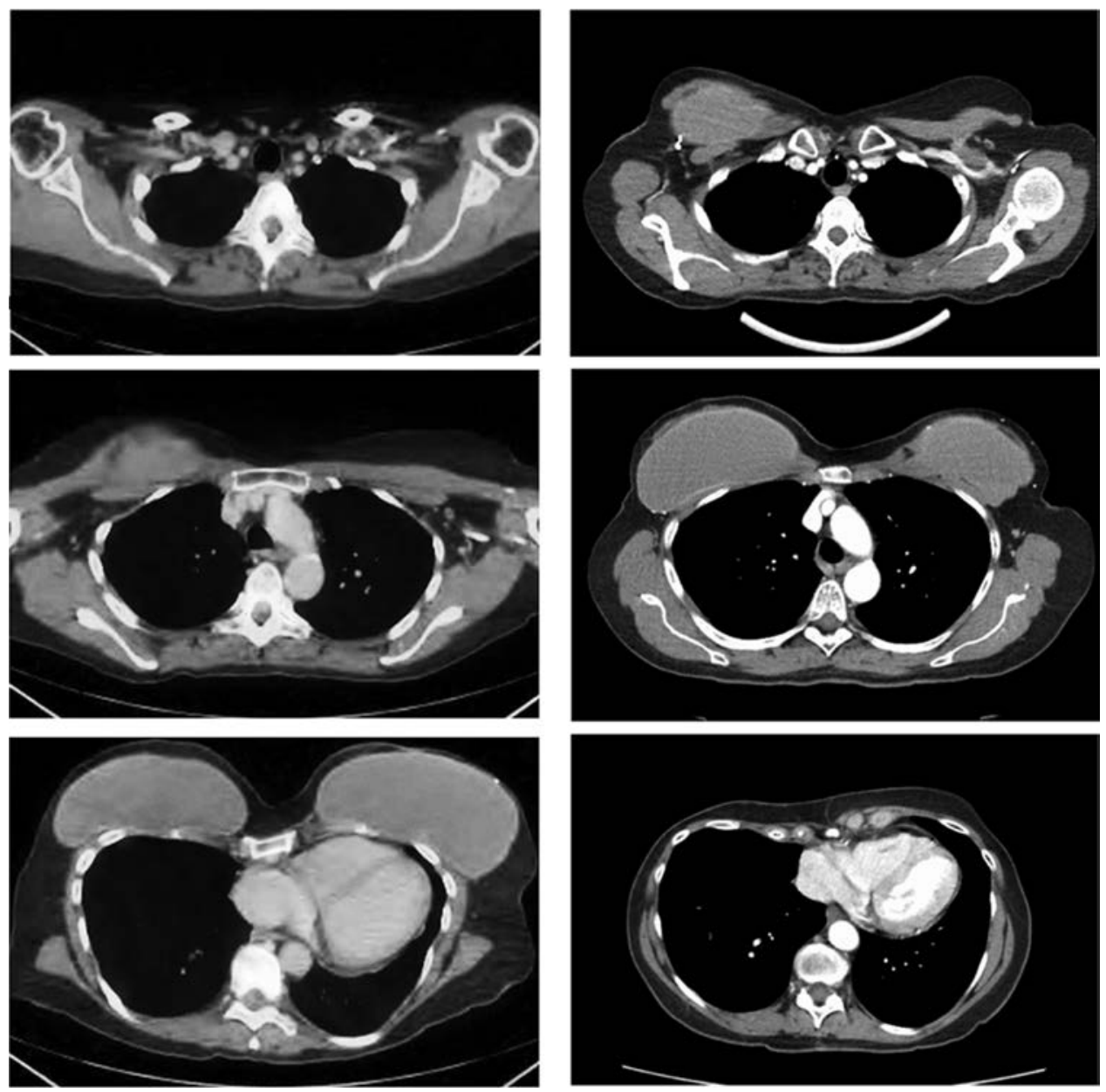

Figure 4. Follow-up scans: representative cuts of positron emission tomography/CT scan at 3 months (left) and CT surveillance at 6 months (right) postoperatively. There was no radiologic evidence of recurrence. 
and faster recovery time. The advantage of the robotic approach with regard to pleural metastasectomy lies in the articulation of the instruments, which allows access to more of the pleural surface that is often limited by the bony thoracic cage in traditional thoracoscopy. In addition, port-site incisions can be used as inflow and outflow tracts for perfusion. There is also less concern about leakage of chemotherapy through the smaller incisions.

We report the first case of robotic-assisted ITH to treat pleural metastasis of ovarian carcinoma and propose that ITH may be a viable treatment option for appropriately selected patients in the future. Larger trials will be necessary to establish the efficacy and safety of ITH for metastatic ovarian cancer and to determine whether there is any impact on recurrence or survival.

\section{References:}

1. Siegel R, Naishadham D, Jemal A. Cancer statistics, 2013. CA Cancer J Clin. 2013;63:11-30.

2. Jemal A, Bray F, Center MM, Ferlay J, Ward E, Forman D. Global cancer statistics. CA Cancer J Clin. 2011;61:69-90.

3. Helm CW. The role of hyperthermic intraperitoneal chemotherapy (HIPEC) in ovarian cancer. Oncologist. 2009; 14: 683-694.
4. Vergote I, Trope CG, Amant F, et al. Neoadjuvant chemotherapy or primary surgery in stage IIIC or IV ovarian cancer. N Engl J Med. 2010;363:943-953.

5. Hess LM, Benham-Hutchins M, Herzog TJ, et al. A metaanalysis of the efficacy of intraperitoneal cisplatin for the frontline treatment of ovarian cancer. Int J Gynecol Cancer. 2007; 17(3):561-570.

6. Bakrin N, Cotte E, Golfier F, et al. Cytoreductive surgery and hyperthermic intraperitoneal chemotherapy (HIPEC) for persistent and recurrent advanced ovarian carcinoma: a multicenter, prospective study of 246 patients. Ann Surg Oncol. 2012;19: 4052-4058.

7. Morgan RJ Jr, Alvarez RD, Armstrong DK, et al. Epithelial ovarian cancer. J Natl Compr Canc Netw. 2011;9:82-113.

8. Witkamp AJ, de Bree E, Van Goethem AR, Zoetmulder FAN Rationale and techniques of intra-operative hyperthermic intraperitoneal chemotherapy. Cancer Treat Rev. 2001;27:365-374.

9. Yellin A, Simansky DA, Paley M, Refaely Y. Hyperthermic pleural perfusion with cisplatin: early clinical experience. Cancer. 2001;92(8):2197-2203.

10. Tilleman TR, Richards WG, Zellos L, et al. Extrapleural pneumonectomy followed by intracavitary intraoperative hyperthermic cisplatin with pharmacologic cytoprotection for treatment of malignant pleural mesothelioma: a phase II prospective study. J Thorac Cardiovasc Surg. 2009;138(2):405-411. 